\title{
Registo médico orientado por problemas em medicina geral e familiar: atualização necessária
}

\author{
Mónica Granja, ${ }^{1-2}$ Conceição Outeirinho
}

\section{RESUMO}

Os registos clínicos são uma componente fundamental dos cuidados médicos. Em medicina geral e familiar é privilegiada a organização dos registos segundo o método proposto por Lawrence Weed: o Registo Médico Orientado por Problemas (RMOP).

É tradicionalmente aceite que o RMOP é constituído por três componentes: dados base, notas clínicas progressivas e lista de problemas. No entanto, não existe um padrão de trabalho definido para a sua utilização e, em Portugal, a bibliografia sobre a utilização do RMOP em Medicina Geral e Familiar carece de atualização.

Pretende-se com este documento sintetizar e compilar a informação disponível, revisitando o método para os médicos de família em exercício e apoiando documentalmente a formação pré e pós-graduada.

Palavras-chave: Medicina geral e familiar; Registos médicos; Registo médico orientado por problemas

\section{INTRODUÇÃO}

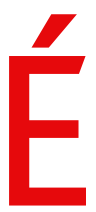

conhecida e reconhecida a importância dos registos clínicos enquanto componente fundamental dos cuidados médicos. ${ }^{1}$ Como suporte informativo, os registos devem refletir o mais corretamente possível o estado de saúde da pessoa e permitir comunicar factos relevantes. Na atividade médica funcionam como auxiliar de memória, permitem a transferência de informação para outras bases de dados e para relatórios médicos e contribuem para articulação de cuidados, auditorias, gestão da prática clinica, formação e investigação. Um registo organizado, para além de permitir uma prática clínica adequada, pode ser considerado, só por si, um documento científico. ${ }^{1}$

Em medicina geral e familiar (MGF), pela abrangência e longitudinalidade de cuidados, é privilegiada a organização dos registos médicos segundo o método proposto por Lawrence Weed nos anos 60 do século $\mathrm{XX}$, mais conhecido por Registo Médico Orientado por Problemas (RMOP). ${ }^{1}$ Como dizia Weed, "a forma como lidamos com a informação determina a forma como pensamos". ${ }^{2}$

O RMOP tem o privilégio de atender aos cinco objetivos operativos definidos por McWhinney. ${ }^{3} \mathrm{Ou}$ seja, permite o acesso rápido aos dados básicos da pessoa, fornece dados contínuos sobre os problemas de saúde, acompa-

1. Médica de Família. Centro de Saúde de S. Mamede Infesta, Unidade Local de Saúde de Matosinhos.

2. Professora auxiliar convidada (Unidades curriculares de Medicina Geral e Familiar I e II). Instituto de Ciências Biomédicas Abel Salazar, Universidade do Porto.

3. Médica de Família. USF Garcia de Orta, ACeS Porto Ocidental. nhando as diferentes consultas, possibilita a obtenção de dados para organizar medidas preventivas e contribui para a formação contínua.

Reconhecem-se ainda outras virtudes do RMOP enquanto facilitador da relação médico-doente, melhoria da performance da equipa de saúde, aumento das oportunidades de intervenção de educação para a saúde e de empowerment da pessoa, o conhecimento do estado de controlo da doença, a possibilidade de caracterizar o perfil de consumo de cuidados de saúde, levando o médico muitas vezes a entender a verdadeira natureza subjacente do problema.

O ordenamento da informação clínica no método de Weed facilita o conhecimento da pessoa e a reflexão sobre os problemas de saúde identificados, levando à tomada de decisões de intervenção. O RMOP é, pois, um sistema de resolução de problemas.

É tradicionalmente aceite que o RMOP é constituído por três componentes: dados base, notas clínicas progressivas e lista de problemas. No entanto, não existe um padrão de trabalho definido para a sua utilização. ${ }^{4}$ Em Portugal encontraram-se dois documentos orientando para a utilização do RMOP em geral. Ambos são antigos (1991 e 2000), de difícil acesso e estão já algo desadaptados à prática atual. ${ }^{5-6}$ Duas publicações mais recentes orientam para a utilização das notas clínicas progressivas na sua estruturação $\mathrm{SOAP}^{7-8}$

Torna-se, assim, pertinente revisitar este método, compilando num só texto a informação dispersa e completando-a. Pretende-se obter um documento-guia prático e que possa apoiar a formação pré e pós-graduada sobre re- 
gistos médicos, bem como ser uma oportunidade de atualização do conhecimento.

\section{Dados base}

Os dados base da pessoa devem incluir, entre outros, a identificação da pessoa, nome e/ou título pelo qual prefere ser tratada, escolaridade, hábitos saudáveis e nocivos, movimentos migratórios, composição do agregado familiar, identificação de recursos sociais e familiares, de eventuais apoios e cuidadores informais, condições de habitação, antecedentes familiares e pessoais, incluindo história familiar, social e laboral e as patologias, com respetivos tratamentos médicos e cirúrgicos, medicação prolongada e sequelas resultantes, incapacidades e grau de dependência/autonomia, história ginecológica e obstétrica nas mulheres, alergias, estado de imunização e realização de rastreios preconizados. Os interesses e atividades e as relações sociais e familiares têm aqui o seu local de registo.

Sendo um conjunto de dados pregressos que, na sua maioria, se preconiza serem colhidos numa primeira consulta, esta não é uma secção estática, devendo sofrer atualizações ou adendas sempre que oportuno. São exemplo comum as mudanças na situação laboral, a ocorrência de novos antecedentes familiares, a alteração de hábitos nocivos, como o tabagismo ou o consumo de álcool, ou mesmo a adoção de hábitos saudáveis.

\section{Notas clínicas progressivas}

As notas clínicas progressivas (ou notas de seguimento) são os registos de cada consulta e organizam-se numa estrutura de quatro itens designada pelo acrónimo SOAP. A abordagem da pessoa numa consulta tem como base a gestão destas notas de seguimento.

OS corresponde a «subjetivo» e inclui os motivos de consulta, interessando registar não só os motivos que a pessoa refere expressamente como tal, mas também aqueles que eventualmente estejam apenas implícitos, nomeadamente aqueles que a pessoa por vezes revela apenas no encerrar da consulta. O contexto também deve ser registado: se é uma consulta com o médico de família (MF), programada ou não programada, se é de intersubstituição e de quem foi a iniciativa (própria pessoa, MF, enfermeiro, outro profissional de saúde, cuidador ou outro). Segue-se a anamnese com esclarecimento e caracterização de eventuais sintomas, queixas e sentimentos (da agenda da pessoa), assim como o resultado da exploração de questões levantadas pelo médico sobre a saúde global restante bem como relativas à agenda do médico. É ainda incluído neste item a explora-

\begin{tabular}{|l|}
$\begin{array}{l}\text { QUADRO I. Conteúdo do item «Subjetivo» das notas } \\
\text { clínicas progressivas }\end{array}$ \\
\hline \multicolumn{1}{c|}{ Subjetivo } \\
Contexto \\
Intersubstituição/outra \\
Consulta programada/outra \\
Iniciativa médica/de enfermagem/outra \\
\hline Fonte da informação \\
\hline Motivos de consulta \\
Expressos / reais \\
\hline Anamnese, incluindo sentimentos \\
Agenda da pessoa \\
Agenda do médico \\
Perspetiva e expectativas da pessoa \\
Recursos identificados pela pessoa \\
\hline
\end{tabular}

ção da perspetiva da pessoa sobre os seus problemas, as suas expectativas e a avaliação que faz dos recursos de que dispõe para resolver os seus problemas (Quadro I) ${ }^{7,9}$

O 0 corresponde a «objetivo». Nesta área devem ser registadas as informações factuais e objetiváveis, relativas à pessoa, como os dados do exame objetivo dirigido à natureza da consulta. O conceito de exame objetivo dirigido, ou orientado, opõe-se ao de exame standard ao ter em conta a pessoa e o tipo de problema ou de vigilância que o traz à consulta. Pode, assim, variar entre um exame mais dirigido (no caso de problemas simples e/ou específicos) e um exame completo (como no exame do recém-nascido) ou no estudo de quadros sistémicos. No $\mathbf{O}$ registam-se ainda resultados de meios complementares de diagnóstico relevantes eventualmente apresentados na consulta, bem como resultados da aplicação de escalas ou questionários. Não sendo um ponto consensual, tem sido recomendado que neste item se inclua, ainda, a informação clínica de outros prestadores (notas de alta de outros níveis de cuidados, relatórios ou registos de outras consultas). ${ }^{5,7}$ É também no $\mathbf{O}$ que faz sentido ser registado, quando relevante, quem acompanha a pessoa à consulta (Quadro II).

O A deve espelhar a avaliação que o médico faz dos problemas da pessoa identificados na consulta em questão. Tal como na lista de problemas, os problemas devem ser registados segundo o seu mais alto grau de resolução no momento. Assim, poderão ser registados diagnósticos bem definidos, problemas de saúde ou ainda sinais ou sintomas a esclarecer. Para cada problema, se aplicável, deve ser especificada a lateralidade, gravidade, grau de controlo e evolução. ${ }^{9}$ Também, sempre que aplicável, podem ser colocadas neste item hipóteses de diagnósticos e diagnósti- 


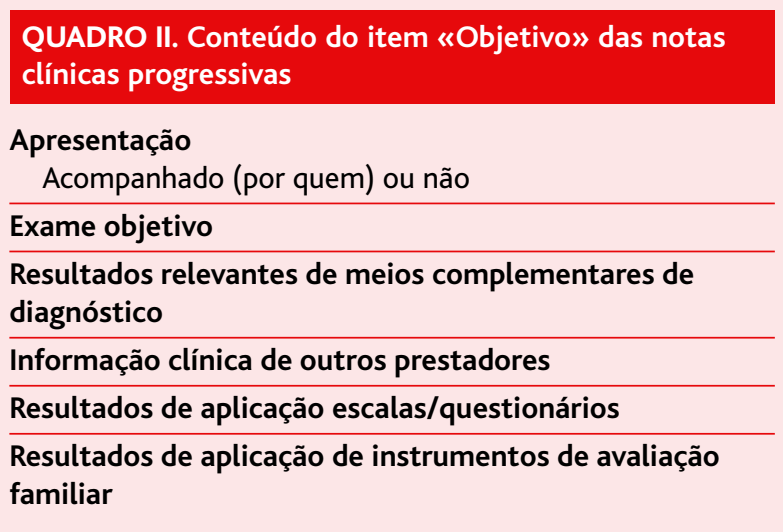

\begin{tabular}{l} 
QUADRO III. Conteúdo do item «Avaliação» das notas \\
clínicas progressivas \\
Avaliação \\
\hline Tipo de vigilância \\
Saúde infantil e juvenil \\
Saúde materna \\
Planeamento familiar \\
Hipertensão arterial \\
Diabetes \\
Outra \\
\hline Problemas identificados na consulta \\
Lateralidade \\
Gravidade \\
Controlo \\
Evolução
\end{tabular}

cos diferenciais. Apenas devem ser listados no A os problemas identificados e abordados na consulta em causa. É a partir do A que se constrói a lista de problemas mas, como veremos adiante, nem todos os problemas do $\mathbf{A}$ da consulta transitam para a lista de problemas da pessoa. No caso de consultas de vigilância, no A cabe também o registo do tipo de vigilância em causa (Quadro III).

No $\mathbf{P}$ deve estar contido o plano de atuação ou intervenção acordado entre médico e doente. Para cada problema devem ser registados os procedimentos e intervenções realizados na consulta e propostos para o imediato o que respeita à investigação diagnóstica, ${ }^{10}$ à terapêutica (farmacológica ou não), os aconselhamentos e intervenções motivacionais, os procedimentos preventivos (primários, secundários e terciários) e a mobilização de recursos necessários (familiares, sociais e institucionais). Os «cuidados antecipatórios» preconizados na vigilância de saúde infantil e juvenil são procedimentos de aconselhamento e devem ser incluídos nes-

\begin{tabular}{|c|c|}
\hline \multicolumn{2}{|c|}{$\begin{array}{l}\text { QUADRO IV. Conteúdo do item «Plano» das notas } \\
\text { clínicas progressivas }\end{array}$} \\
\hline \multicolumn{2}{|r|}{ Plano } \\
\hline & Plano de estudo (investigação diagnóstica) \\
\hline & $\begin{array}{l}\text { Plano terapêutico } \\
\text { Farmacológico ou não }\end{array}$ \\
\hline \multirow{8}{*}{$\begin{array}{l}\text { Prazo } \\
\text { Curto } \\
\text { Médio } \\
\text { Longo } \\
\end{array}$} & Plano educacional \\
\hline & Plano preventivo \\
\hline & Recursos a mobilizar \\
\hline & Referenciações \\
\hline & Certificados e outros documentos emitidos \\
\hline & $\begin{array}{l}\text { Consultas agendadas } \\
\text { Com médico de família } \\
\text { Com outros prestadores }\end{array}$ \\
\hline & Respostas a e-mails \\
\hline & Notas / reflexões do médico \\
\hline
\end{tabular}

te item. Além das intervenções previstas a curto prazo, devem ainda ser registadas as intervenções previstas ou recomendadas a médio e a longo prazo (Quadro IV).

No $\mathbf{P}$, o registo das intervenções a implementar para cada problema identificado em A deve ser sistematizado e hierarquizado de acordo com a carga de doença ou grau de importância para a pessoa e os recursos disponíveis ou mobilizáveis. Registam-se, também, todos os procedimentos e intervenções a desenvolver, no âmbito da abordagem global (biopsicossocial) de saúde da pessoa.

Cabe ainda neste item o registo de referenciações a outros prestadores, de documentos emitidos (certificados de incapacidade temporária, relatórios, etc.) e de consultas agendadas para reavaliação, vigilância de saúde ou noutros níveis de cuidados. As notas e reflexões do médico sobre a pessoa e a sua circunstância, assim como eventuais falhas a corrigir na consulta seguinte também têm lugar no P. Hoje em dia, com a possibilidade de comunicação não presencial ( $e$-mail e telefone) entre médicos e doentes, o feedback dado pelo médico à pessoa por estes canais deve também ser registado no $\mathbf{P}$.

\section{Lista de problemas}

A lista de problemas é um resumo dos problemas relevantes da pessoa e é referida por Weed como sendo uma espécie de índice dos restantes registos. ${ }^{1,4}$ Por este motivo, é considerada, isoladamente, como o componente mais importante do RMOP. Não há uma definição consensual sobre o que constitui um problema de saúde. Um problema pode 
ser considerado "tudo aquilo que preocupa o médico, o doente, ou ambos" ${ }^{11}$ ou tudo o que for importante nos cuidados da pessoa. ${ }^{3}$ Os problemas devem ser listados ao mais alto nível de resolução a cada momento, podendo coexistir sintomas ou sinais para os quais (ainda) não exista uma explicação ou diagnóstico preciso, problemas familiares (como doenças ou conflitos), problemas psicológicos ou sociais (como a pobreza ou o desemprego) e diagnósticos já estabelecidos. ${ }^{1,5}$ Assim, a lista de problemas é uma componente dinâmica, devendo ser atualizada sempre que problemas são resolvidos ou identificados de novo e sempre que um dos problemas listados é revisto e passa a integrar um novo problema ou diagnóstico. ${ }^{1}$

\begin{tabular}{|c|c|c|c|c|c|}
\hline \multicolumn{6}{|c|}{$\begin{array}{l}\text { QUADRO V. Local de registo dos resultados da aplicação dos instrumentos de } \\
\text { avaliação familiar }\end{array}$} \\
\hline \multirow{2}{*}{ Instrumentos de avaliação familiar } & \multirow{2}{*}{$\begin{array}{l}\text { Dados } \\
\text { básicos }\end{array}$} & \multicolumn{4}{|c|}{ Notas clínicas progressivas } \\
\hline & & $\mathbf{S}$ & 0 & A & $\mathbf{P}$ \\
\hline Genograma & $\mathrm{x}$ & & $\mathrm{X}$ a) & & \\
\hline Ciclo de vida familiar de Duvall & $\mathrm{x}$ & & & & \\
\hline Biopatografia / Linha de vida de Medalie & $x$ & & & & \\
\hline Psicofigura de Mitchell & & & $x$ & & \\
\hline Círculo familiar de Thrower & & & $\mathrm{x}$ & & \\
\hline Apgar familiar de Smilkstein & & & $\mathrm{x}$ & & \\
\hline Risco familiar de Segóvia-Dryer & & & $x$ & & \\
\hline Risco familiar de García-Gonzalez & & & $\mathrm{x}$ & & \\
\hline Escala de readaptação social de Holmes e Rahe & & & $\mathrm{x}$ & & \\
\hline Dinâmica familiar de Minuchin & & & $\mathrm{x}$ & & \\
\hline Eco-mapa & & & $x$ & & \\
\hline Modelo de Olsen & & & $\mathrm{X}$ & & \\
\hline
\end{tabular}

a) Em situações de reavaliação perante os critérios de Janet-Christie-Seely.

Cada problema listado deve ser designado de modo completo (incluindo, se aplicável, etiologia, estadio, lateralidade e grau de controlo), consistente e rigoroso. Existem recomendações para que os sistemas informáticos não forcem a classificação dos problemas da lista ${ }^{4}$ e que disponham de possibilidade de notas em texto livre. ${ }^{1}$

A mera classificação/codificação dos problemas da pessoa, sem a sua descrição, é redutora, porquanto informa sobre a inclusão da pessoa numa entidade nosológica mas não documenta de forma adequada o problema de saúde no indivíduo em particular. ${ }^{9}$

Para cumprir a sua função de índice ou de resumo nem todos os problemas da consulta devem transitar para a lista de problemas, devendo ser excluídos os problemas $m i$ nor, isolados e/ou autolimitados. Este tipo de problemas é apenas registado no A da consulta em que surgir e poderá eventualmente ser arquivado enquanto episódio de cuidados (funcionalidade de algumas aplicações informáticas).

Os problemas que devem constar da lista são as doenças crónicas ou recidivantes, as doenças com mau prognóstico, os problemas que condicionaram tratamentos cirúrgicos, internamentos ou incapacidades, bem como aqueles que, ainda que resolvidos, possam condicionar a saúde ou as decisões terapêuticas futuras. A decisão sobre que problemas devem integrar ou não a lista obedece ao juízo clínico do médico e é por vezes difícil de tomar. Na dúvida sobre a impor- tância de um novo problema pode a decisão ser adiada e revista posteriormente. Existem recomendações para que sejam criadas localmente regras quanto ao tipo de problemas a incluir na lista, assim como a uniformização da terminologia a usar, de modo a tornar coerentes os registos de doentes que circulam entre diferentes prestadores. ${ }^{4}$ Se existir esta coerência interprofissional, pode dizer-se que quanto maior a lista de problemas maior a complexidade de um doente.

Os problemas da lista são, por fim, classificados como «ativos» ou «passivos», conforme, respetivamente, afetem a pessoa à data ou se encontrem resolvidos, mas tendo o potencial de voltar a afetar a pessoa ou de condicionar decisões clínicas. Também esta classificação dos problemas é dinâmica e um problema passivo num dado momento pode tornar-se ativo mais tarde (e vice-versa).

É sugerido porWeed que cada problema da lista seja numerado por ordem de importância e seja depois ligado às notas clínicas progressivas. Esta numeração, se bem que útil quando coexistem múltiplos problemas, na prática pode ser difícil de concretizar. Há problemas que desaparecem, porque resolvidos ou integrados noutros. A própria ordem de importância pode ser difícil de estabelecer e, por vezes, a listagem por ordem cronológica pode ser mais útil. As listas de problemas devem ser periodicamente revistas, em função da complexidade de cada doente. São momentos chave para esta atualização o primeiro e o úl- 
timo passos da consulta, ${ }^{12}$ a elaboração de relatórios clínicos e de cartas de referenciação, a receção de informação de outros níveis de cuidados e qualquer momento de reavaliação da situação clínica da pessoa.

\section{Avaliação familiar}

A inclusão dos resultados dos métodos de avaliação familiar nos registos clínicos não tem uma localização pré-definida. Quando há indicação para a utilização destes métodos, na maioria dos casos devem ser registados no $\mathbf{O}$, enquanto resultado da aplicação de uma escala ou questionário. As exceções poderão ser o genograma, a linha de vida de Medalie e o Ciclo de vida familiar de Duvall, cuja localização natural será nos dados base (Quadro V).

\section{CONCLUSÃO}

A importância dos registos médicos na prestação de cuidados de saúde é reconhecida. O RMOP é o sistema de registos adotado pela MGF e foi definido há mais de 50 anos. Em Portugal, a bibliografia sobre a utilização do RMOP em MGF carece de atualização.

Embora a versatilidade do RMOP permitisse a sua utilização em sistemas informáticos, as plataformas desenvolvidas em Portugal apresentam ainda vários constrangimentos e dificuldades na sua completa aplicação tal como foi concebido por Weed. ${ }^{1}$ Neste documento revisita-se o RMOP e compila-se a informação sobre o conteúdo de cada um dos seus componentes para apoiar os MF em exercício e a formação pré e pós-graduada.

SeWeed dizia "Nós não cuidamos de registos, cuidamos de pessoas, mas os registos não podem ser separados dos cuidados", ${ }^{2}$ pode-se acrescentar que se a MGF e os MF não cuidarem dos seus registos é a prestação de cuidados que está comprometida.

\section{REFERÊNCIAS BIBLIOGRÁFICAS}

1. Weed LL. Medical records that guide and teach. N Engl J Med. 1968;278(11): 593-600.

2. Weed L. Larry Weed's 1971 internal medicine grand rounds [Youtube].VisualDx; 2012 [cited 2017 Mar 15]. Available from: https://www.youtube.com/watch?v= qMsPXSMTPFI

3. McWhinney IR, Freeman T. Records. In: McWhinney IR, Freeman T, editors. A textbook of family medicine. 3rd ed. New York: Oxford University Press; 2009. p. 375-8.

4. Simons SM, Cillessen FH, Hazelzet JA. Determinants of a successful problem list to support the implementation of the problem-oriented medical record according to recent literature. BMC Med Inform Decis Mak. 2016;16:102.

5. Sousa JC. Sistemas de anotação clínica. In: Associação Portuguesa dos Médicos de Clínica Geral, editor. Manual de medicina geral e familiar: versão 0.01 [Internet]. Lisboa:APMGF; 2000 [cited 2017 Jan 22].Available from: https://www.mgfamiliar.net/ MMGF/textos/12/10_texto.html

6. Caeiro RT. Registos clínicos em medicina familiar. Lisboa: Instituto de Clínica Geral da Zona Sul; 1991.

7. Santiago LM. Contributo para a melhoria dos registos em consulta. Rev Ordem Médicos. 2015;31(161):60-2

8. Queiroz MJ. SOAP revisitado [SOAP revisited]. Rev Port Clin Geral. 2009;25(2):2217. Portuguese

9. Pinto D. O que classificar nos registos clínicos com a Classificação Internacional de Cuidados Primários? [What should we code in health records with the International Classification of Primary Care?]. Rev Port Med Geral Fam. 2014;30(5):328-34. Portuguese

10. Barreto JV, Paiva P. O registo clínico orientado por problemas [The problem oriented clinical record]. Rev Soc Port Med Int. 2008;15(3):201-6. Portuguese

11. Hurst JW. The art and science of presenting a patient's problems (as an extension of the Weed system). Arch Intern Med. 1971;128(3):463-5.

12. Ramos $V$. A consulta em 7 passos: execução e análise crítica de consultas em medicina geral e familiar [Internet]. Lisboa: VFBM Comunicação; 2008. ISBN 9789898160225.Available from: http://www.apmgf.pt/websites/apmgf/A\%20Consulta\%207\%20passos.pdf

\section{CONFLITOS DE INTERESSE}

Os autores declaram não ter quaisquer conflitos de interesse.

\section{ENDEREÇO PARA CORRESPONDÊNCIA}

Mónica Granja

E-mail: monicagranja66@gmail.com

Recebido em 01-04-2017

Aceite para publicação em 10-01-2018

\section{ABSTRACT}

\section{PROBLEM-ORIENTED MEDICAL RECORD IN FAMILY PRACTICE: A NECESSARY UPDATE}

Medical records are a key component of health care delivery. In family practice, the preferred approach to structure medical records is based on the method proposed by Lawrence Weed', the 'Problem-oriented medical record'.

It is generally accepted that the 'Problem-oriented medical record' comprises three components: baseline data, progressive clinical notes and list of problems. Nevertheless, there is a lack of standards on how to use these sections and, in Portugal, the bibliography on the use of 'Problem-oriented medical record' needs updating.

This document aims to synthesise and compile the information available, revisiting Weed's method for practising family physicians and supporting pre- and postgraduate training.

Keywords: Family practice; Medical records; Problem-oriented medical records 